\title{
Force Fields for Molecular Modeling of Sarin and its Simulants: DMMP and DIMP
}

\author{
Alina Emelianova, ${ }^{\dagger, \S}$ Elizaveta A. Basharova, ${ }^{\ddagger}, \S$ Andrei L. Kolesnikov, " Evaristo \\ Villaseco Arribas, ${ }^{\dagger}$ Ella V. Ivanova, ${ }^{\dagger}$ and Gennady Y. Gor ${ }^{*, \dagger}$ \\ $\dagger$ Otto H. York Department of Chemical and Materials Engineering, \\ New Jersey Institute of Technology, University Heights, Newark, NJ 07102, USA \\ $\ddagger$ Institut für Theoretische Physik, Universität Leipzig, Brüderstr. 15, 04103 Leipzig, \\ Germany \\ 【Institut für Nichtklassische Chemie e.V., Permoserstr. 15, 04318 Leipzig, Germany \\ $\S$ Contributed equally to this work \\ E-mail: gor@njit.edu

\section{Supporting Information}

TABLE S1: Partial charges of DIMP united atoms calculated using various levels of theory (in $e$ ), and based on the partial atomic charges of sarin and DMMP. For rightmost column shows the sources for each of the charges for the TraPPE model.

\begin{tabular}{l|r|r|r|r|l}
\hline atom & HF & MP2 & DFT & TraPPE & Source \\
\hline $\mathrm{P}$ & 1.520 & 1.450 & 1.290 & 1.36 & adjusted \\
$\mathrm{O}=$ & -0.850 & -0.830 & -0.740 & -0.80 & DMMP \\
$\mathrm{O}-$ & -0.685 & -0.660 & -0.600 & -0.63 & sarin \\
$\mathrm{CH}_{3}(-\mathrm{P})$ & -0.100 & -0.100 & -0.090 & -0.14 & DMMP \\
$\mathrm{CH}_{3}(-\mathrm{CH})$ & -0.120 & -0.100 & -0.100 & -0.10 & sarin \\
$\mathrm{CH}$ & 0.640 & 0.600 & 0.570 & 0.62 & sarin \\
\hline
\end{tabular}

The calculations with TraPPE force field with combined charges give the density of DIMP at $298 \mathrm{~K}$ of $1003 \pm 4 \mathrm{~kg} / \mathrm{m}^{3}$ and $998 \pm 5 \mathrm{~kg} / \mathrm{m}^{3}(298 \mathrm{~K})$ from MC and MD simulations, 
respectively, which does not deviate much from the results based on the HF charges. Fig. S1 shows a comparison of the experimental liquid densities ${ }^{1}$ and prediction by the TraPPE force field with the two sets of charges by both MC and MD: both predictions by MC and MD were similar to each other with only a slight deviation from experimental data. Fig. S2a shows the comparison of coexistence curves and Clausius-Clapeyron plots for vapor pressure simulated with different charges. The figure demonstrates a negligible effect of the partial charges with the highest deviation between the densities in approximately $20 \mathrm{~kg} / \mathrm{m}^{3}$ for both liquid and gas phases at $600 \mathrm{~K}$.

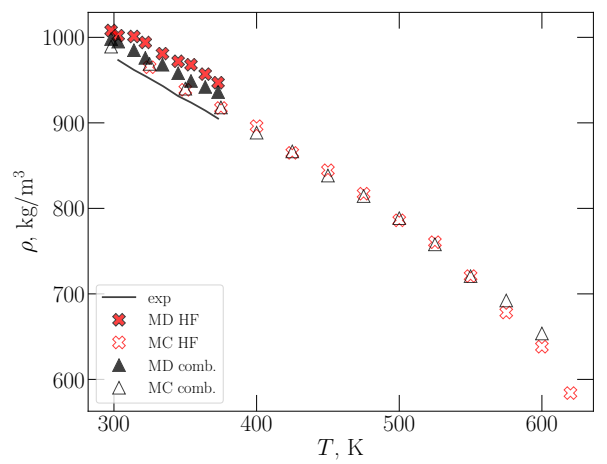

FiguRE S1: Liquid densities of DIMP predicted with MC and MD simulations based on TraPPE force field with partial charges obtained by HF method (crosses) and combined from TraPPE (triangles) along with the experimental data ${ }^{1]}$ (solid line).

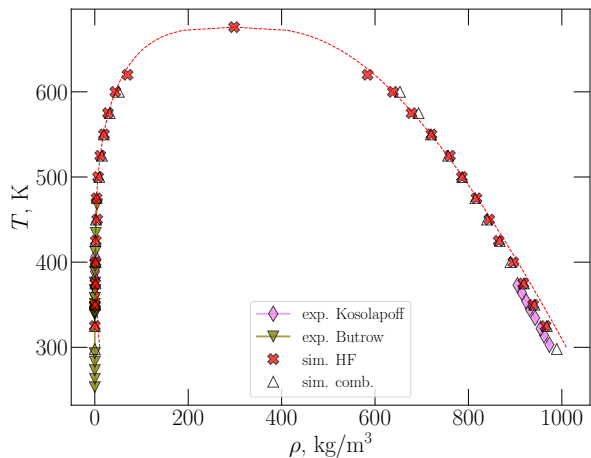

(a)

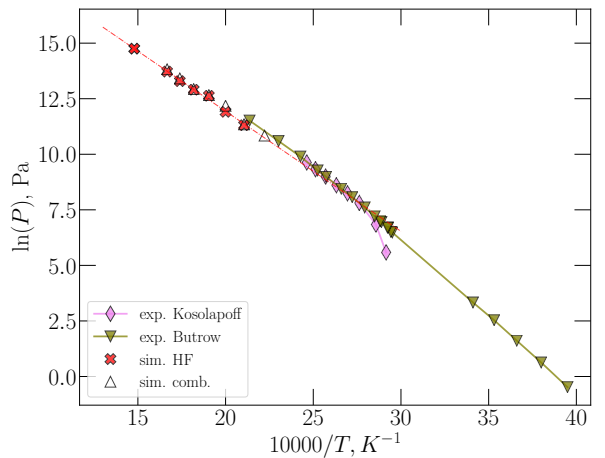

(b)

FIgURE S2: Vapor-liquid coexistence curves based on TraPPE force fields along with the fit of critical point and experimental data (a) and the corresponding Clausius-Clapeyron plot along with the experimental data and the linear fit of the simulation data (b) for DIMP based on simulations with charges from HF method (crosses) and combined from TraPPE (triangles). 
TABLE S2: Vapor-liquid equilibrium data for DMMP from GEMC simulation.

\begin{tabular}{|c|c|c|c|}
\hline$T, \mathrm{~K}$ & $\rho_{\text {gas }}, \mathrm{kg} / \mathrm{m}^{3}$ & $\rho_{\text {liq }}, \mathrm{kg} / \mathrm{m}^{3}$ & $P$, atm \\
\hline \multicolumn{4}{|c|}{ DMMP (VN2004) } \\
\hline$\overline{350}$ & 0.02 & 1115.03 & 0.01 \\
\hline 400 & 0.22 & 1069.10 & 0.06 \\
\hline 425 & 0.68 & 1045.69 & 0.19 \\
\hline 450 & 1.13 & 1020.44 & 0.33 \\
\hline 475 & 2.20 & 994.65 & 0.67 \\
\hline 500 & 4.06 & 968.16 & 1.27 \\
\hline 525 & 6.66 & 940.12 & 2.17 \\
\hline 550 & 10.83 & 912.33 & 3.56 \\
\hline 575 & 17.19 & 881.38 & 5.69 \\
\hline 600 & 25.35 & 846.90 & 8.33 \\
\hline 625 & 36.88 & 810.54 & 12.02 \\
\hline 650 & 54.55 & 768.36 & 16.88 \\
\hline 675 & 81.08 & 722.24 & 23.68 \\
\hline \multicolumn{4}{|c|}{ DMMP (TraPPE) } \\
\hline$\overline{375}$ & 0.19 & 1107.32 & 0.10 \\
\hline 400 & 0.55 & 1058.80 & 0.15 \\
\hline 425 & 1.50 & 1035.37 & 0.41 \\
\hline 450 & 2.70 & 1002.46 & 0.79 \\
\hline 475 & 5.65 & 973.20 & 1.65 \\
\hline 500 & 8.27 & 941.70 & 2.75 \\
\hline 525 & 13.93 & 906.49 & 4.41 \\
\hline 550 & 21.27 & 874.89 & 6.75 \\
\hline 575 & 33.87 & 837.95 & 10.65 \\
\hline 600 & 48.56 & 793.61 & 14.95 \\
\hline 625 & 66.56 & 724.36 & 20.10 \\
\hline \multicolumn{4}{|c|}{ DMMP (Ruffley et al.) } \\
\hline$\overline{300}$ & 0.0017 & 1148.96 & \\
\hline 325 & 0.08 & 1121.81 & \\
\hline 350 & 0.23 & 1099.29 & 0.05 \\
\hline 375 & 0.42 & 1071.30 & 0.10 \\
\hline 400 & 1.21 & 1025.94 & 0.31 \\
\hline 450 & 5.73 & 962.17 & 1.57 \\
\hline 500 & 17.29 & 887.26 & 4.88 \\
\hline 525 & 25.99 & 834.23 & 9.03 \\
\hline 550 & 43.00 & 794.53 & 11.72 \\
\hline 575 & 60.51 & 723.80 & \\
\hline 600 & 108.64 & 600.01 & \\
\hline
\end{tabular}


TABLE S3: Vapor-liquid equilibrium data for sarin and DIMP from GEMC simulation.

\begin{tabular}{|c|c|c|c|}
\hline$T, \mathrm{~K}$ & $\rho_{\text {gas }}, \mathrm{kg} / \mathrm{m}^{3}$ & $\rho_{\text {liq }}, \mathrm{kg} / \mathrm{m}^{3}$ & $P$, atm \\
\hline \multicolumn{4}{|c|}{ sarin (TraPPE) } \\
\hline$\overline{325}$ & 0.001 & 1075.26 & \\
\hline 350 & 0.50 & 1044.43 & \\
\hline 400 & 1.99 & 983.85 & \\
\hline 440 & 5.21 & 932.63 & 1.27 \\
\hline 480 & 12.89 & 877.87 & 3.27 \\
\hline 520 & 26.89 & 817.93 & 6.86 \\
\hline 560 & 49.65 & 739.58 & 12.46 \\
\hline 600 & 95.93 & 638.14 & 21.83 \\
\hline \multicolumn{4}{|c|}{ DIMP (TraPPE) } \\
\hline$\overline{350}$ & 3.84 & 938.63 & \\
\hline 375 & 0.35 & 917.39 & \\
\hline 400 & 0.57 & 896.09 & \\
\hline 425 & 1.38 & 864.90 & \\
\hline 450 & 1.97 & 844.56 & \\
\hline 475 & 5.24 & 817.14 & 0.81 \\
\hline 500 & 9.39 & 786.02 & 1.49 \\
\hline 525 & 14.50 & 760.43 & 3.06 \\
\hline 550 & 21.84 & 720.84 & 4.03 \\
\hline 575 & 31.62 & 678.21 & 5.92 \\
\hline 600 & 44.90 & 638.18 & 9.09 \\
\hline 620 & 70.00 & 584.00 & \\
\hline \multicolumn{4}{|c|}{ DIMP (TraPPE, comb.) } \\
\hline$\overline{350}$ & 0.76 & 941.22 & \\
\hline 375 & 1.43 & 918.91 & \\
\hline 400 & 1.61 & 890.12 & \\
\hline 425 & 1.38 & 866.87 & \\
\hline 450 & 2.57 & 839.78 & 0.51 \\
\hline 475 & 4.06 & 814.70 & 0.85 \\
\hline 500 & 9.53 & 786.63 & 1.95 \\
\hline 525 & 15.13 & 756.58 & 3.15 \\
\hline 550 & 19.25 & 719.89 & 4.13 \\
\hline 575 & 31.71 & 693.28 & 6.67 \\
\hline 600 & 51.27 & 653.45 & 10.07 \\
\hline
\end{tabular}

\section{References}

(1) Kosolapoff, G. Vapour pressures and densities of some lower alkylphosphonates. J. Chem. Soc.(Resumed) 1955, 2964-2965. 